\title{
College Mental Health Before and During the COVID-19 Pandemic: Results From a Nationwide Survey
}

\author{
Hanjoo Kim ${ }^{1} \oplus$. Gavin N. Rackoff ${ }^{2}$. Ellen E. Fitzsimmons-Craft ${ }^{3} \cdot$ Ki Eun Shin $^{4} \cdot$ Nur Hani Zainal $^{2}$. Jeremy T. Schwob ${ }^{2}$. \\ Daniel Eisenberg ${ }^{5} \cdot$ Denise E. Wilfley $^{3} \cdot$ C. Barr Taylor ${ }^{6,7} \cdot$ Michelle G. Newman $^{2}$
}

Accepted: 12 June 2021 / Published online: 19 June 2021

(c) The Author(s), under exclusive licence to Springer Science+Business Media, LLC, part of Springer Nature 2021

\begin{abstract}
Background The COVID-19 pandemic could affect college students' mental health. We examined screening rates for psychological disorders before and during the pandemic.

Methods Undergraduates were surveyed before $(n=3643)$ or during the pandemic $(n=4970)$. Logistic regression adjusting for participant demographics was conducted.

Results Frequencies of depression [OR 1.32, 95\% CI $(1.17,1.48)$ ], alcohol use disorder [OR 1.70, 95\% CI $(1.50,1.93)]$, bulimia nervosa/binge-eating disorder [OR 1.54, 95\% CI $(1.28,1.85)]$, and comorbidity [OR 1.19, 95\% CI $(1.04,1.35)]$ were greater during (vs. before) the pandemic. Frequencies of posttraumatic stress disorder were lower during the pandemic [OR $0.86,95 \% \mathrm{CI}(0.75,0.98)]$. The upward trend in alcohol use disorder was stronger among women than men [OR $1.47,95 \%$ CI $(1.18,1.83)]$. The upward trend in depression was stronger among Black students than White students [OR 1.72, 95\% CI $(1.19,2.49)]$. Anxiety disorders, insomnia, anorexia nervosa, and suicidality showed no significant trends.

Conclusions Depression, alcohol use disorder, bulimia nervosa/binge-eating disorder, and comorbidity were higher, whereas posttraumatic stress disorder was lower during the pandemic. Women and Black students could face especially heightened risk for alcohol use disorder and depression, respectively, during the pandemic.
\end{abstract}

Keywords COVID-19 $\cdot$ Pandemic $\cdot$ College students $\cdot$ Psychological disorders

Hanjoo Kim and Gavin N. Rackoff are the Co-first authors

Michelle G. Newman

mgn1@psu.edu

1 Department of Psychiatry, Michigan Medicine, Ann Arbor, MI, USA

2 Department of Psychology, The Pennsylvania State University, 371 Moore Building, University Park, PA, USA

3 Department of Psychiatry, Washington University School of Medicine, St. Louis, MO, USA

4 Department of Psychology, Teachers College, Columbia University, New York City, NY, USA

5 Department of Health Policy and Management, Fielding School of Public Health, University of California at Los Angeles, Los Angeles, CA, USA

6 Department of Psychiatry and Behavioral Sciences, Stanford University School of Medicine, Stanford, CA, USA

7 Center for m2 Health, Palo Alto University, Palo Alto, CA, USA

\section{Introduction}

Since the first case of COVID-19 was recorded in November 2019 , the disease has spread rapidly, resulting in over 3 million deaths worldwide as of April 23rd, 2021 (World Health Organization, 2020). The pandemic has introduced mental health risk factors including the threat of illness and death, isolation, and economic uncertainty. Accordingly, there has been interest in the impact of the pandemic on psychological conditions including emotional disorders, eating disorders, and alcohol misuse (Adhanom Ghebreyesus, 2020; Clay \& Parker, 2020; Fernandez-Aranda et al., 2020). For example, adults in Australia reported greater binge and restrictive eating during the pandemic than before the pandemic (Phillipou et al., 2020), and adults in Poland reported drinking more alcohol during than before the pandemic (Sidor \& Rzymski, 2020). Moreover, two studies in the United States documented increased rates of psychological distress during the pandemic compared to before the pandemic (American Psychological Association, 2020; Twenge \& Joiner, 2020). 
Thus, initial data suggest that the COVID-19 pandemic has elevated risk for several mental health problems among adults.

College students' mental health could also be severely affected by the pandemic. US college students were asked to leave campuses in the middle of the 2019-2020 academic year, disrupting coursework and causing losses to independence and social support. Such disruptions could have had an adverse impact given college students' heightened psychological vulnerability. For example, epidemiological surveys conducted before the pandemic documented a high 12-month prevalence of psychological disorders (31.1\%) among college students (Auerbach et al., 2018), and rates of anxiety, depression, and suicidality increased in this population throughout the 2010s (Duffy et al., 2019). Furthermore, approximately $75 \%$ of lifetime psychological disorders have onset during young adulthood (Kessler et al., 2007). Thus, college students are a vulnerable group who may develop mental health problems during the pandemic.

In fact, evidence suggests an adverse psychological impact of the pandemic on college-aged young adults. In Italy, young adults had higher anxiety and distress during the pandemic than respondents in their fifties or older (Casagrande et al., 2020). Similarly, Chinese adults in college had higher stress and anxiety during the pandemic than those not in college (Wang et al., 2020). However, these studies were limited by a lack of comparison data collected before the pandemic, which made it difficult to attribute college students' mental health problems to the pandemic vs. existing vulnerabilities. A stronger approach to study population-level trends in psychological disorders would involve inviting everyone in the cohort of interest to complete identical validated screening measures during the two periods of interest (i.e., shortly before and during the pandemic) and comparing the two groups (Busfield, 2012). One study using such a method in Greece found increased depression and suicidality among college students during the pandemic, relative to norms from 2012 and earlier (Kaparounaki et al., 2020), though it remains unclear whether these changes can be attributed to the pandemic vs. mental health trends of increased rates over time in the 2010s (Duffy et al., 2019). Overcoming this limitation, one study documented increases in alcohol use among U.S. students throughout March 2020, though changes in other mental health problems were not addressed (Lechner et al., 2020). Gaps in the literature call for examining a comprehensive range of psychological conditions among college students using data from immediately before and during the pandemic.

The current study examined the prevalence of positive screens for various psychological disorders among first- and second-year undergraduates across eight U.S. schools, who provided data either a few months before or after the onset of the U.S. COVID-19 outbreak. We compared rates for clinical levels of depression, anxiety disorders, posttraumatic stress disorder, eating disorders, insomnia, suicidality, and alcohol use disorder, as well as their comorbidity, before and during the pandemic. We also tested whether gender identity, race, and sexual orientation moderated differences in rates of positive psychological disorder screens over time. We hypothesized that the prevalence and comorbidity of psychological disorders would be higher during the pandemic than before the pandemic. We had no specific hypotheses regarding whether demographic factors would moderate these associations. The current study presents comprehensive data on the COVID-19's impact on U.S. college students' mental health to guide policymaking and wellness initiatives.

\section{Methods}

\section{Participants}

First- or second-year undergraduate students at 4-year colleges and universities completed a mental health screening survey between October 2019 and May 2020. The screening survey was sent to all first- and second-year students at participating institutions and was conducted to determine eligibility for later enrollment in a randomized controlled trial (ClinicalTrials.gov-Harnessing Mobile Technology to Reduce Mental Health NCT04162847); thus, participants were recruited from the general population of all first- and second-year undergraduates at the colleges/universities. All data for the present study were collected prior to selection for the randomized controlled trial or intervention delivery.

A total of 11,576 students accessed the survey. Participants were excluded if they did not provide informed consent $(n=1568)$, were under 18 years old $(n=168)$, were not first- or second-year undergraduates $(n=656)$, participated in January or February 2020, when awareness of the coronavirus in the U.S. was mixed $(n=342)$, exited the survey before reporting on any psychological disorders $(n=216)$, or failed an attention check that asked participants to choose a specific response $(n=13)$. The resulting sample included 8613 students who participated either during the pre-pandemic period ( $n=3643$; October 7, 2019 through December $1,2019)$ or during the pandemic period $(n=4970$; March 2, 2020 through May 9, 2020). Students were recruited from different schools in geographically diverse states across the pre-pandemic period (West, $n=1358$; Midwest, $n=1151$; South, $n=879$; East, $n=255$; four total schools) and the pandemic period (West, $n=213$; Midwest, $n=1051$; South, $n=979$; East, $n=2727$; four total schools). Each student participated once, and thus no students participated in both periods. Please refer to Table 1 for demographics. 
Table 1 Participant demographic characteristics

\begin{tabular}{|c|c|c|c|c|c|}
\hline & \multicolumn{2}{|c|}{$\begin{array}{l}\text { Pre-pandemic } \\
(n=3643)\end{array}$} & \multicolumn{2}{|c|}{$\begin{array}{l}\text { Pandemic } \\
(n=4970)\end{array}$} & \multirow[t]{2}{*}{$\begin{array}{l}\chi^{2} \\
(d f)\end{array}$} \\
\hline & $n$ & $\%$ & $n$ & $\%$ & \\
\hline \multicolumn{6}{|l|}{ Sex assigned at birth } \\
\hline Male & 977 & 26.83 & 1505 & 30.33 & \multirow[t]{3}{*}{$13.22 * *(2)$} \\
\hline Female & 2662 & 73.11 & 3456 & 69.65 & \\
\hline Intersex & 2 & 0.05 & 1 & 0.02 & \\
\hline \multicolumn{6}{|l|}{ Gender identity } \\
\hline Male & 967 & 26.62 & 1500 & 30.22 & \multirow[t]{3}{*}{ 45.56*** (2) } \\
\hline Female & 2535 & 69.80 & 3387 & 68.23 & \\
\hline Trans, non-conforming, or self-identify & 130 & 3.58 & 77 & 1.55 & \\
\hline \multicolumn{6}{|l|}{ Race } \\
\hline White & 2496 & 72.01 & 3551 & 74.79 & \multirow[t]{6}{*}{$110.04 * * *(5)$} \\
\hline Black or African American & 306 & 8.83 & 261 & 5.50 & \\
\hline Asian & 344 & 9.92 & 683 & 14.39 & \\
\hline American Indian or Alaskan native & 23 & 0.66 & 22 & 0.46 & \\
\hline Native Hawaiian or Pacific Islander & 13 & 0.38 & 11 & 0.23 & \\
\hline Multiracial & 284 & 8.19 & 220 & 4.63 & \\
\hline \multicolumn{6}{|l|}{ Ethnicity } \\
\hline Hispanic & 424 & 11.67 & 511 & 10.33 & \multirow[t]{2}{*}{$3.82(1)$} \\
\hline Non-Hispanic & 3210 & 88.33 & 4434 & 89.67 & \\
\hline \multicolumn{6}{|l|}{ Sexual orientation } \\
\hline Heterosexual & 2592 & 71.37 & 4030 & 81.43 & \multirow[t]{2}{*}{$119.32 * * *(1)$} \\
\hline $\begin{array}{l}\text { Lesbian, gay, bisexual, queer, questioning, } \\
\text { or self-identify }\end{array}$ & 1040 & 28.63 & 919 & 18.57 & \\
\hline \multicolumn{6}{|l|}{ Year in school } \\
\hline First-year & 2235 & 61.35 & 3051 & 61.39 & \multirow[t]{2}{*}{$0.00(1)$} \\
\hline Second-year & 1408 & 38.65 & 1919 & 38.61 & \\
\hline
\end{tabular}

$n=8613$; Pre-pandemic: October 7, 2019 through December 1, 2019, Pandemic: March 2, 2020 through May 9, 2020; Chi-square statistics reflect likelihood ratio chi-square values for the association between time (pre-pandemic vs. pandemic) and demographic characteristics; $* * p<0.01$, *** $p<0.001$; Statistically significant results are bolded $(p<0.05)$

\section{Procedures}

Researchers emailed invitations to complete a survey on well-being to all first- and second-year students at participating schools. Emails informed students that, based on their responses, they may be eligible for a subsequent study involving random assignment to conditions designed to support mental health. Emails included a link to an online screening survey. Participating students were entered into a raffle for a $\$ 100$ prize. The study was approved by the institutional review board of all authors' universities and administrators at each participating school.

\section{Measures}

Generalized anxiety disorder (GAD), social anxiety disorder (SAD), and panic disorder were assessed using the Generalized Anxiety Disorder Questionnaire, 4th edition, with 0.82 specificity and 0.89 sensitivity (Newman et al., 2002), Social
Phobia Diagnostic Questionnaire, with 0.95 specificity and 0.57 sensitivity (Moore et al., 2014), and Panic Disorder Self-Report, with 1.00 specificity and 0.89 sensitivity (Newman et al., 2006), respectively. These measures assess all diagnostic criteria based on the Diagnostic and Statistical Manual of Mental Disorders, 5th edition (American Psychiatric Association, 2013). Participants screened positive for a disorder if they endorsed all diagnostic criteria.

Posttraumatic stress disorder (PTSD) was assessed using the Primary Care PTSD Screen (Prins et al., 2003). Participants screened positive for probable PTSD if they scored three or higher, which demonstrated sensitivity of 0.78 and specificity of 0.89 (Prins et al., 2003).

Major depressive disorder (MDD) was assessed using the Patient Health Questionnaire-9 (PHQ-9) (Kroenke \& Spitzer, 2002). Participants screened positive for probable MDD if they scored 10 or higher, with sensitivity of 0.88 and specificity of 0.85 (Manea et al., 2012). Suicidal ideation was assessed using item 9 of the PHQ-9, which asked 
how much respondents thought about hurting themselves or that they would be better off dead in the past two weeks. Responses of 1 (several days) or higher screened positive for suicidal ideation.

Anorexia nervosa (AN) and bulimia or binge-eating disorder (BN/BED) were assessed using the Stanford-Washington University Eating Disorder Screen (Graham et al., 2019). Participants screened positive for probable AN if they scored 59 or higher on a weight/shape concerns scale and had a current body mass index $\leq 18.45$, based on self-reported height and weight. Participants screened positive for probable BN/ BED if they did not screen positive for AN and reported objective binge eating, self-induced vomiting, or diuretic or laxative use six or more times in the past 3 months. These criteria have been used in prior online screening studies (Fitzsimmons-Craft et al., 2019).

Insomnia was assessed using the Insomnia Severity Index (Morin et al., 2011). Participants screened positive for probable insomnia if they scored 15 or higher, a cutoff with sensitivity of 0.78 and specificity of 1.00 (Morin et al., 2011).

Alcohol use disorder (AUD) was assessed using the Alcohol Use Disorders Identification Test Consumption (AUDITC) (Bush et al., 1998). To identify probable AUD, we used the cutoff of 4 or higher for participants assigned male at birth and 3 or higher for participants assigned female or intersex. This system had 0.88 sensitivity and 0.75 specificity for males and 0.87 sensitivity and 0.85 specificity for females (Bradley et al., 2007).

\section{Analytic Procedures}

To examine demographic characteristic (i.e., sex assigned at birth, gender identity, race, ethnicity, sexuality, year in school, geographic region) differences between the prepandemic and pandemic cohorts, we conducted multinomial logistic regression analyses comparing proportions of the cohorts belonging to each demographic group. We also compared age across the pre-pandemic and pandemic cohorts using an independent samples $t$ test.

For the main analyses, we performed binary logistic regression comparing proportions of the sample screening positive for each psychological disorder across time (prepandemic vs. pandemic). Additionally, to examine comorbidity, we performed binary logistic regression comparing the proportion of participants screening positively for two or more psychological disorders (vs. 0 or 1 disorder) at each time point. We also conducted exploratory analyses testing demographic factors (gender identity, race, and sexual orientation) as moderators of the association between time and psychological disorder prevalence. Time, demographic factors, and two-way interactions between time and demographic factors were modeled as predictors of proportions of positive screens and comorbidity. Significant omnibus interactions were probed by examining simple contrast effects comparing the association between time and psychological disorder prevalence for participants across specific groups. To adjust for possible confounding, all demographic characteristics found to differ across samples in the preliminary analyses were included as covariates in the main analyses (D'Onofrio et al., 2020). Analyses were conducted in $R$ 3.6.2.

\section{Results}

The highest percentage of missing data was $13.42 \%$ for $\mathrm{BN} /$ BED. This was due to nonresponse and inability to score non-numeric responses (e.g., "I don't know," "sometimes") to questions asking about the frequency of binging and purging. For other variables, missing data ranged from 0.13 to $10.26 \%$ and were due only to nonresponse. Missing data were handled using pairwise deletion.

Please see Table 1 for participant demographic characteristics. Multinomial logistic regression analyses revealed significant differences across cohorts in terms of sex assigned at birth, $\chi^{2}(2)=13.22, p=0.001$, gender identity, $\chi^{2}(2)=45.56, p<0.001$, race, $\chi^{2}(5)=110.04, p<0.001$, sexual orientation $\chi^{2}(1)=119.32, p<0.001$, and geographic region, $\chi^{2}(3)=3,127.84, p<0.001$. An independent samples $t$-test also indicated that the pandemic cohort $\left(M_{\text {age }}=19.50\right.$, $\left.S D_{\text {age }}=3.30\right)$ was significantly older than the pre-pandemic cohort $\left(M_{\text {age }}=18.85, S D_{\text {age }}=2.08\right), \beta=0.65, S E=0.06$, $t(8611)=10.45, p<0.001$. There were no significant differences across cohorts in proportions of participants identifying as Hispanic, $\chi^{2}(1)=3.82, p=0.051$, or proportion of first vs. second year students, $\chi^{2}(1)=0.00, p=0.972$. In light of these differences, sex assigned at birth, gender identity, sexual orientation, race, geographic region, and age were included as covariates in subsequent analyses. Please note that the addition of Hispanic ethnicity and year in school as covariates did not change the results, and therefore only the demographic variables that differed significantly across cohorts were included as covariates in the analyses reported here.

Table 2 shows the proportions of participants meeting screening criteria for each psychological disorder across time. Compared to the pre-pandemic period, there was a significantly greater proportion of participants meeting criteria for MDD in the pandemic period, $\chi^{2}(1)=21.67, p<0.001$, OR $1.32,95 \%$ CI $[1.17,1.48]$. Odds of positive screens for AUD were also significantly greater in the pandemic period, $\chi^{2}(1)=67.26, p<0.001$, OR $1.70,95 \%$ CI $[1.50,1.93]$. The same pattern was found for BN/BED, $\chi^{2}(1)=20.83$, $p<0.001$, OR $1.54,95 \%$ CI $[1.28,1.85]$. Rates of psychological comorbidity were also higher during the pandemic period than during the pre-pandemic period, $\chi^{2}(1)=6.83$, 
Table 2 Prevalence of psychological conditions before and during the COVID-19 pandemic periods

\begin{tabular}{|c|c|c|c|c|}
\hline & & $\begin{array}{l}\text { Pre-pan- } \\
\text { demic } \\
(n=3643)\end{array}$ & $\begin{array}{l}\text { Pandemic } \\
(n=4970)\end{array}$ & $\chi^{2}(d f=1)$ \\
\hline \multirow[t]{2}{*}{ GAD } & Non-clinical & $2498(71.0 \%)$ & $3486(72.1 \%)$ & 0.19 \\
\hline & Clinical & $1022(29.0 \%)$ & $1347(27.9 \%)$ & \\
\hline \multirow[t]{2}{*}{ SAD } & Non-clinical & $2793(83.3 \%)$ & $3962(85.4 \%)$ & 2.20 \\
\hline & Clinical & $561(16.7 \%)$ & $678(14.6 \%)$ & \\
\hline \multirow[t]{2}{*}{ Panic } & Non-clinical & $2964(87.4 \%)$ & $4204(89.8 \%)$ & 3.39 \\
\hline & Clinical & $427(12.6 \%)$ & $477(10.2 \%)$ & \\
\hline \multirow[t]{2}{*}{ PTSD } & Non-clinical & $2186(67.5 \%)$ & $3218(71.1 \%)$ & $5.46 *$ \\
\hline & Clinical & $1052(32.5 \%)$ & $1306(28.9 \%)$ & \\
\hline \multirow[t]{2}{*}{ AN } & Non-clinical & $3159(98.0 \%)$ & $4432(98.1 \%)$ & 0.15 \\
\hline & Clinical & $64(2.0 \%)$ & $88(1.9 \%)$ & \\
\hline \multirow[t]{2}{*}{ BN/BED } & Non-clinical & $2668(89.3 \%)$ & $3826(85.6 \%)$ & $20.83 * * *$ \\
\hline & Clinical & $320(10.7 \%)$ & $643(14.4 \%)$ & \\
\hline \multirow[t]{2}{*}{ Insomnia } & Non-clinical & $2647(82.4 \%)$ & $3655(81.0 \%)$ & 0.17 \\
\hline & Clinical & $567(17.6 \%)$ & $860(19.0 \%)$ & \\
\hline \multirow[t]{2}{*}{ AUD } & Non-clinical & $2072(64.2 \%)$ & $2631(58.3 \%)$ & $67.26 * * *$ \\
\hline & Clinical & $1154(35.8 \%)$ & $1878(41.7 \%)$ & \\
\hline \multirow[t]{2}{*}{ MDD } & Non-clinical & $2217(61.0 \%)$ & $2693(54.3 \%)$ & $21.67 * * *$ \\
\hline & Clinical & $1418(39.0 \%)$ & $2270(45.7 \%)$ & \\
\hline \multirow[t]{2}{*}{ SI } & Non-clinical & $2867(78.8 \%)$ & $3976(80.1 \%)$ & 0.00 \\
\hline & Clinical & $770(21.2 \%)$ & 989 (19.9\%) & \\
\hline \multirow[t]{2}{*}{ Comorbidity } & $\begin{array}{l}\text { 0-1 Disor- } \\
\text { ders }\end{array}$ & $1482(52.1 \%)$ & $2070(48.0 \%)$ & $6.83 * *$ \\
\hline & $2+$ Disorders & $1362(47.9 \%)$ & $2240(52.0 \%)$ & \\
\hline
\end{tabular}

$n=8613$; Pre-pandemic: October 7, 2019 through December 1, 2019, Pandemic: March 2, 2020 through May 9, 2020

$G A D$ Generalized anxiety disorder, $S A D$ Social anxiety disorder, Panic Panic disorder, PTSD Posttraumatic stress disorder, AN Anorexia nervosa, $B N / B E D$ Bulimia nervosa/binge eating disorder, $A U D$ Alcohol use disorder, MDD Major depressive disorder, SI Suicidal ideation

$\chi^{2}$ statistics reflect Wald test values for the logistic regression association between time (pre-pandemic vs. pandemic) and psychological conditions, adjusting for age, sex assigned at birth, gender identity, race, sexual orientation, and geographic region; probabilities in table are unadjusted for demographics; $* p<0.05, * * p<0.01, * * * p<0.001$; Statistically significant results are bolded $(p<0.05)$

$p=0.009, \mathrm{OR} 1.19,95 \%$ CI $[1.04,1.35]$. In contrast, rates of PTSD were significantly lower during the pandemic period than during the pre-pandemic period, $\chi^{2}(1)=5.46$, $p=0.019$, OR $0.86,95 \%$ CI $[0.75,0.98]$. Thus, in line with the expectation of greater rates of psychological disorders during the pandemic, the pandemic cohort had higher rates of MDD, AUD, BN/BED, and comorbid psychological disorders. However, contrary to expectation, the pandemic cohort also had lower rates of PTSD.

Unlike the aforementioned psychological disorders, there was not a significant difference across time in rate of positive screens for SAD, $\chi^{2}(1)=2.20, p=0.138$, OR $0.89,95 \%$
Table 3 Interactions among demographic factors and time in predicting probabilities of psychological conditions

\begin{tabular}{lccl}
\hline & $\begin{array}{l}\text { Gender } \times \text { Time } \\
\chi^{2}(d f=2)\end{array}$ & $\begin{array}{l}\text { Race } \times \text { Time } \\
\chi^{2}(d f=5)\end{array}$ & $\begin{array}{l}\text { Sexual- } \\
\text { ity } \times \text { Time } \\
\chi^{2} \\
(d f=1)\end{array}$ \\
\hline GAD & 4.47 & 7.88 & 0.83 \\
SAD & 1.73 & 2.67 & 2.56 \\
Panic & 3.65 & 7.00 & 1.84 \\
PTSD & 4.26 & 3.12 & 0.80 \\
AN & 0.88 & 6.81 & 1.89 \\
BN/BED & 3.79 & 3.90 & 0.00 \\
Insomnia & 2.12 & 1.95 & 0.13 \\
AUD & $\mathbf{1 1 . 2 9 * *}$ & 0.98 & 0.37 \\
MDD & 0.32 & $\mathbf{1 1 . 5 2 *}$ & 1.23 \\
SI & 1.66 & 5.70 & 0.20 \\
Comorbidity & 2.97 & 4.52 & 0.66 \\
\hline
\end{tabular}

$n=8613$; Pre-pandemic: October 7, 2019 through December 1, 2019, Pandemic: March 2, 2020 through May 9, 2020

$G A D$ Generalized anxiety disorder, $S A D$ Social anxiety disorder, Panic Panic disorder, PTSD Posttraumatic stress disorder, AN Anorexia nervosa, $B N / B E D$ Bulimia nervosa/binge eating disorder, $A U D$ Alcohol use disorder, $M D D$ Major depressive disorder, SI Suicidal ideation

$\chi^{2}$ statistics reflect Wald test values for the logistic regression interaction between time (pre-pandemic vs. pandemic) and demographic factors predicting psychological conditions, adjusting for age, sex assigned at birth, gender identity, race, sexual orientation, and geographic region; $* p<0.05, * * p<0.01$; Statistically significant results are bolded $(p<0.05)$

CI $[0.75,1.04]$, panic disorder, $\chi^{2}(1)=3.39, p=0.066$, OR $0.84,95 \%$ CI $[0.71,1.01]$, GAD, $\chi^{2}(1)=0.19, p=0.664$, OR $0.97,95 \%$ CI $[0.85,1.11]$, insomnia, $\chi^{2}(1)=0.17$, $p=0.684$, OR $0.97,95 \%$ CI $[0.83,1.13], \mathrm{AN}, \chi^{2}(1)=0.15$, $p=0.700$, OR $0.92,95 \% \mathrm{CI}[0.62,1.39]$, and suicidal ideation, $\chi^{2}(1)=0.00, p=0.988$, OR $1.00,95 \%$ CI $[0.87,1.15]$. Thus, for these conditions, there was no observed difference from before to during the pandemic.

Results from moderator analyses are shown in Table 3. There was a significant interaction between time and gender in predicting AUD, $\chi^{2}(2)=11.29, p=0.004$. Simple contrasts indicated that, compared to participants who identified as male (estimated probability $=27.58 \%$ before pandemic vs. $32.91 \%$ during pandemic) the upward trend in rate of AUD was significantly larger among participants who identified as female (estimated probability $=34.40 \%$ before pandemic vs. $49.74 \%$ during pandemic), $\chi^{2}(1)=11.53, p=0.001$, OR $1.47,95 \%$ CI $[1.18,1.83]$, but the upward trend was not significantly different among participants who had other gender identities (estimated probability $=17.08 \%$ before pandemic vs. $23.98 \%$ during pandemic), $\chi^{2}(1)=0.19, p=0.666$, OR $1.19,95 \% \mathrm{CI}[0.54,2.60]$. Thus, the analyses found that the 
higher rate of AUD during (vs. before) the pandemic was more pronounced among women than among men.

There was also a significant interaction between time and race in predicting rates of MDD, $\chi^{2}(5)=11.52, p=0.042$. Simple contrasts indicated that, compared to participants who identified as White (estimated probability $=39.53 \%$ before pandemic vs. $43.76 \%$ during pandemic), the difference over time in rate of MDD was significantly larger among participants who identified as Black (estimated probability $=33.58 \%$ before pandemic vs. $50.86 \%$ during pandemic), $\chi^{2}(1)=8.23, p=0.004$, OR $1.72,95 \%$ CI $[1.19,2.49]$. However, simple contrasts also indicated that the difference over time in rate of MDD relative to White participants was not significantly larger or smaller among participants who identified as Asian (estimated probability $=36.83 \%$ before pandemic vs. $47.04 \%$ during pandemic), $\chi^{2}(1)=2.60, p=0.107$, OR $1.28,95 \%$ CI [0.95, 1.73], American Indian or Alaskan Native (estimated probability $=40.20 \%$ before pandemic vs. $52.63 \%$ during pandemic), $\chi^{2}(1)=0.27, p=0.602$, OR 1.39 , 95\% CI [0.41, 4.87], Native Hawaiian or other Pacific Islander (estimated probability $=51.78 \%$ before pandemic vs. $64.11 \%$ during pandemic), $\chi^{2}(1)=0.15, p=0.695$, OR $1.40,95 \%$ CI $[0.27,7.99]$, or Multiracial (estimated probability $=39.73 \%$ before pandemic vs. $51.14 \%$ during pandemic), $\chi^{2}(1)=2.13$, $p=0.144$, OR $1.33,95 \%$ CI $[0.91,1.97]$. Thus, the analyses suggested that compared to students identifying as White, among students not identifying as White, only those identifying as Black showed a greater upward trend in rates of MDD during (vs. before) the pandemic.

\section{Discussion}

Our nationwide data on first- and second-year undergraduates revealed a complex picture of mental health trends among college students during the COVID-19 pandemic. Screening rates of MDD, BN/BED, AUD, and comorbid psychological disorders were greater during the early months of the COVID-19 pandemic compared to late 2019. In contrast, rates of PTSD were lower during the early months of the COVID-19 pandemic compared to late 2019. Moderator analyses found that the upper trend in rates for AUD from pre-pandemic to during the pandemic was significantly higher for participants identifying as female than for participants identifying as male. Furthermore, the upper trend in rates of MDD from pre-pandemic to during the pandemic was significantly higher for participants identifying as Black than for participants identifying as White. There were no differences in rates of panic disorder, SAD, GAD, insomnia, AN, or suicidal ideation during (vs. before) the pandemic. We offer several potential explanations for these findings.
The higher frequency of positive screens for MDD during the pandemic could have emerged due to health and economic risks posed by the pandemic, paired with the loss of outlets for physical activity (e.g., recreation centers) and social engagement (e.g., student organizations). Cognitive-behavioral theory suggests that losses in positively reinforcing, pleasurable activities contribute to the risk for depression (Ferster, 1973), and this could be a mechanism contributing to the greater rate of MDD during the pandemic. Beyond the population-wide risk factors posed by the pandemic, some person-specific risk factors (e.g., loss of a loved one) could have also led individual students to screen positively for MDD. Importantly, the moderator analyses also found that the upward trend in rates of MDD during (vs. before) the pandemic was larger among students identifying as Black than students identifying as White. Thus, the onset of the pandemic appeared to especially impact depression among Black students relative to White students. Although it will be crucially important to collect data on mechanisms contributing to this difference, it deserves noting that Black Americans have suffered especially high rates of COVID-19 infection and mortality (Abedi et al., 2020) and duration of unemployment during the pandemic (Couch et al., 2020). Thus, illness and economic risk factors for depression were likely more severe among Black students. Black Americans have also been historically underserved by mental healthcare services within both the student and general population (Eisenberg et al., 2011; Lê Cook et al., 2017), which could have amplified risk for depression during the pandemic. A multitude of systemic influences likely contribute to racial disparities in COVID-19's mental health impact, and the present findings call for close examination of colleges' and universities' support of Black students during the pandemic and beyond.

Beyond MDD, positive screens for two conditions characterized by poor health behavior, BN/BED and AUD, were also more common during the pandemic than before the pandemic. Higher rates of BN/BED might be explained by stockpiling of groceries, restrictions in exercising, and difficulty abstaining from food while at home (Touyz et al., 2020). Likewise, higher rates of AUD during the pandemic could have resulted from greater downtime with alcohol stocked at home. Such interpretations are consistent with reports of heightened eating disorder behavior and alcohol use among adults during the pandemic (Phillipou et al., 2020; Sidor \& Rzymski, 2020), as well as theory and evidence that excessive availability of food and alcohol cues can contribute to binge eating and drinking (Jansen, 1998; Weitzman et al., 2003). Moderator analyses indicated that the pandemic was associated with an especially high elevation in AUD among students identifying as female, relative to students identifying as male. This finding might have arisen because young women are more likely than young 
men to regulate emotions using alcohol (Peltier et al., 2019), and women in the present study's sample might have used alcohol to cope during the pandemic at greater rates than men. This interpretation is in line with a recent report that found a stronger association between COVID-19-related distress and alcohol use among women than men (Rodriguez et al., 2020). It is important to note that living situation characteristics (e.g., food and alcohol kept at home) and severity of pandemic-related distress likely varied substantially across individuals. Thus, research should seek to identify the unique pathways by which the pandemic could affect risk for BN/BED and AUD in the general student population and among women specifically.

In contrast to other disorders, positive screens for PTSD were less common during (vs. before) the pandemic. This finding is somewhat surprising given the possibility for COVID-19 infection and bereavement to impact posttraumatic stress symptoms among those who experience these events (Gallagher et al., 2021). However, lower rates of PTSD in the present study's sample might have emerged because stay-at-home measures enabled avoidance of some situations that could trigger trauma-related distress. Thus, whereas it is possible that some individuals experienced traumatic stress related to the COVID-19 pandemic, the population-wide trend could reflect lower rates of PTSD because staying at home can reduce the frequency of encounters with cues associated with other traumatic experiences. Substantial evidence suggests that avoidance is related to lower PTSD-linked distress, at least in the short term (Pittig et al., 2018). Importantly, the temporary symptom relief resulting from avoidance may negatively reinforce avoidance, making PTSD symptoms less amenable to change in the long term (Pittig et al., 2018). Thus, examining the psychological effects of stay-at-home measures and assessing PTSD after in-person activities resume will have significant public health implications.

In contrast to other conditions, screening rates of GAD, $\mathrm{SAD}$, panic disorder, insomnia, $\mathrm{AN}$, and suicidality did not differ across the pre-pandemic and pandemic cohorts. Whereas the health and economic risks posed by the pandemic might have exacerbated anxiety symptoms characteristic of GAD, SAD, and panic disorder, other changes brought about by the pandemic could have offset these influences. For example, many universities introduced pass/fail grading systems during the Spring 2020 semester (Burke, 2020), which could have reduced academic pressure and buffered a potential increase in anxiety symptoms. Relatedly, whereas the lifestyle changes introduced by the pandemic could have adversely affected eating behaviors, social distancing efforts might have also reduced the saliency of social pressures to attain a thin body shape (e.g., Garner \& Garfinkel, 1980), leading to an absence in population-wide differences in AN rates. Likewise, although daily routine changes resulting from the switch to online learning could have adversely affected sleep for some students (Bootzin \& Perlis, 2011), other students might have found greater time to sleep with the elimination of morning commutes to class, limiting population-wide differences in rates of insomnia during (vs. before) the pandemic. Finally, the absence of a difference in rates of suicidality is surprising given the higher rates of MDD during the pandemic. However, because not all conditions demonstrated higher rates of mental health problems over time, it is possible that college students' overall mental health did not undergo severe enough changes to increase suicidality from before to during the pandemic. Each of these mental health problems has a complex set of determinants, and future research might identify subgroups of students who experienced changes in these conditions with the onset of the pandemic.

Although the pattern of differences over time for each condition was variable, there were a higher rate of participants screening positively for comorbid (i.e., two or more) conditions during the pandemic than before the pandemic. Given the high disability and illness cost associated with having comorbid psychological disorders (Andrews et al., 2001), this finding suggests the possibility of an especially adverse population-wide impact of the pandemic. Higher rates of comorbidity during the pandemic could have resulted from the introduction of transdiagnostic risk factors that increased risk for multiple, co-occurring conditions (e.g., isolation, economic hardship; Grant et al., 2003; Lasgaard et al., 2011). The finding calls for more research on risk factors contributing to comorbid conditions during the pandemic, as well as research on transdiagnostic interventions that can simultaneously address multiple disorders in the student population.

This study had a number of limitations. First, we limited our analyses to first- and second-year students at 4-year institutions, raising the possibility that the sample was not representative of all college students (e.g., more senior students or students at 2-year institutions). Additionally, this study lacked repeated measurements of the same participants from before to during the pandemic; therefore, we cannot infer within-person changes in psychopathology across time. However, our population-based recruitment approach, in which all first- and second-year students at participating schools were invited to complete identical screening measures shortly before and during the pandemic, is a strong method for studying population-wide mental health trends (Busfield, 2012). Additionally, there were differences across the samples in terms of participants' school geographic region and personal demographics. By adjusting for all demographic characteristics that differed across cohorts, our analytical approach strengthened the inferences that could be drawn from comparisons across the cohorts. Therefore, despite these limitations, we believe our sample can provide 
valuable information about COVID-19-related mental health trends of U.S. undergraduates.

Beyond the above-mentioned limitations of the dataset, additional methodological limitations also deserve note. Our study used self-report measures, which can lead to higher prevalence estimates than diagnostic interviews (Thombs et al., 2018). Thus, it is possible that lower rates would have been found had we used structured interviews. Relatedly, the recruitment strategy (which informed students that they might be eligible for a subsequent study involving conditions designed to support mental health) could have led to greater participation among distressed students. It should be noted, however, that the self-report measures used in the present study have been psychometrically validated and demonstrate strong sensitivity and specificity relative to interview-based diagnoses. The positive screen rates in the present study were also similar to those of epidemiological studies of college students conducted before the pandemic. For example, Auerbach et al. (2018) found a 31.1\% 12-month prevalence of self-reported mental disorders among college students between 2014 and 2017 using questionnaires aligning with diagnostic criteria. More recently, Duffy et al. (2019) found that $41.1 \%$ of surveyed students in the 2017-2018 school year met screening criteria for MDD using the same screening criteria as the present study, and Lipson, Phillips, Winquist, Eisenberg, and Lattie (2021) found that 55.0\% of 4-year college students met criteria for at least one mental health problem based on validated screening questionnaires between 2016 and 2019. Given the similarity of positive screen rates in the present study and prior epidemiological investigations, it is unlikely that our recruitment or assessment methods inflated estimated rates of mental health problems beyond overall trends for self-report measures. Furthermore, any biases resulting from the recruitment and assessment strategies would affect both cohorts in the present study and would therefore not affect inferences about differences in rates of mental health problems over time. Thus, we believe the results provide a valid picture of student population-wide trends in mental health problems during the pandemic.

We close our discussion by highlighting several important directions for future research. First, the pandemic has continued evolving since Spring 2020, when students in the present study participated. Therefore, it will be important to examine whether and how rates of mental disorders changed in more recent months of the pandemic, as well as how students' mental health could change as the pandemic dwindles with increasing vaccination rates. Relatedly, the study lacked specific information on COVID-19-related stress (e.g., loss of loved ones, contamination fears), as well as additional individual difference factors (e.g., whether participants lived alone, with friends, or with families) that could help explain the pattern of findings and identify subpopulations at elevated risk for mental health problems. Incorporating such measures is crucial to identify mediators and moderators of the pandemic's mental health impact. Finally, whereas the present study identified racial and gender differences in risk for heightened rates of certain mental disorders during the pandemic, it will be just as important for future research to identify personal and community-level factors associated with resilience during the pandemic among Black students, female students, and the broader student population. Such research is critical to providing a complete picture of mental health in the context of the pandemic, as well as to guide initiatives to support students.

\section{Conclusions}

The COVID-19 pandemic's onset may have had a complex set of influences on psychological disorders in first- and secondyear college students. Relative to a cohort of students who provided data in Fall 2019, students who provided data in the early months of the pandemic had higher rates of MDD, BN/ BED, AUD, and psychological comorbidity. However, rates of PTSD were lower during (vs. before) the pandemic. Students identifying as female were at especially high risk for elevated rates of AUD during the pandemic, and students identifying as Black were at especially high risk for elevated rates of MDD during the pandemic. These results provide a picture of COVID-19's initial impact on college students and highlight portions of the student population with unmet mental health needs during the pandemic. Understanding students' mental health during the pandemic is urgently needed for better mental healthcare in the current academic year and beyond. We hope these results will call attention to the need for improved mental health services for college students and spur additional investigations of the pandemic's continued impact on mental health.

Funding The current study was funded by the National Institutes of Health (Grant No. 5R01MH115128-03).

\section{Declarations}

Conflict of Interest Hanjoo Kim, Gavin N. Rackoff, Ellen E. Fitzsimmons-Craft, Ki Eun Shin, Nur Hani Zainal, Jeremy T. Schwob, Daniel Eisenberg, Denise E. Wilfley, C Barr Taylor, Michelle G. Newman declare that they have no conflict of interest.

Ethical Approval Ethics approval was obtained from the IRB of each data collection site.

Informed Consent Informed consent was obtained from all individual participants included in the study.

Animal Rights No animal studies were carried out by the authors for this article. 


\section{References}

Abedi, V., Olulana, O., Avula, V., Chaudhary, D., Khan, A., Shahjouei, S., Li, J., \& Zand, R. (2020). Racial, economic, and health inequality and COVID-19 infection in the United States. Journal of Racial and Ethnic Health Disparities. https://doi.org/10.1007/ s40615-020-00833-4

Adhanom Ghebreyesus, T. (2020). Addressing mental health needs: An integral part of COVID-19 response. World Psychiatry, 19, 129-130. https://doi.org/10.1002/wps.20768

American Psychiatric Association. (2013). Diagnostic and statistical manual of mental disorders (DSM-5) (5th ed.). American Psychiatric Association.

American Psychological Association. (2020). Stress in the Time of COVID-19. Stress in America ${ }^{\mathrm{TM}}$ Survey. Retrieved from https:// www.apa.org/news/press/releases/stress/2020/report

Andrews, G., Henderson, S., \& Hall, W. (2001). Prevalence, comorbidity, disability and service utilisation. Overview of the Australian national mental health survey. British Journal of Psychiatry, 178, 145-153. https://doi.org/10.1192/bjp.178.2.145

Auerbach, R. P., Mortier, P., Bruffaerts, R., Alonso, J., Benjet, C., Cuijpers, P., Demyttenaere, K., Ebert, D. D., Green, J. G., Hasking, P., Murray, E., Nock, M. K., Pinder-Amaker, S., Sampson, N. A., Stein, D. J., Vilagut, G., Zaslavsky, A. M., Kessler, R. C., \& WHO WMH-ICS Collaborators. (2018). WHO world mental health surveys international college student project: Prevalence and distribution of mental disorders. Journal of Abnormal Psychology, 127, 623-638. https://doi.org/10.1037/abn0000362

Bootzin, R. R., \& Perlis, M. L. (2011). Stimulus control therapy. In M. Perlis, M. Aloia, \& B. Kuhn (Eds.), Behavioral treatments for sleep disorders. Academic Press.

Bradley, K. A., DeBenedetti, A. F., Volk, R. J., Williams, E. C., Frank, D., \& Kivlahan, D. R. (2007). AUDIT-C as a brief screen for alcohol misuse in primary care. Alcoholism, Clinical and Experimental Research, 31, 1208-1217. https://doi.org/10.1111/j.15300277.2007.00403.x

Burke, L. (2020). \#PassFailNation, Inside Higher Ed

Busfield, J. (2012). Challenging claims that mental illness has been increasing and mental well-being declining. Social Science and Medicine, 75, 581-588. https://doi.org/10.1016/j.socscimed.2012. 02.053

Bush, K., Kivlahan, D. R., McDonell, M. B., Fihn, S. D., \& Bradley, KAa. (1998). The AUDIT alcohol consumption questions (AUDIT-C): An effective brief screening test for problem drinking. Archives of Internal Medicine, 158, 1789-1795. https://doi. org/10.1001/archinte.158.16.1789

Casagrande, M., Favieri, F., Tambelli, R., \& Forte, G. (2020). The enemy who sealed the world: Effects quarantine due to the COVID-19 on sleep quality, anxiety, and psychological distress in the Italian population. Sleep Medicine. https://doi.org/10.1016/j. sleep.2020.05.011

Clay, J. M., \& Parker, M. O. (2020). Alcohol use and misuse during the COVID-19 pandemic: A potential public health crisis? Lancet Public Health, 5, e259. https://doi.org/10.1016/S2468-2667(20) 30088-8

Couch, K. A., Fairlie, R. W., \& Xu, H. (2020). Early evidence of the impacts of COVID-19 on minority unemployment. Journal of Public Economics, 192, 104287. https://doi.org/10.1016/j.jpube co. 2020.104287

D’Onofrio, B. M., Sjolander, A., Lahey, B. B., Lichtenstein, P., \& Oberg, A. S. (2020). Accounting for confounding in observational studies. Annual Review of Clinical Psychology, 16, 25-48. https:// doi.org/10.1146/annurev-clinpsy-032816-045030
Duffy, M. E., Twenge, J. M., \& Joiner, T. E. (2019). Trends in mood and anxiety symptoms and suicide-related outcomes among U.S. undergraduates, 2007-2018: Evidence from two national surveys. Journal of Adolescent Health, 65, 590-598. https://doi.org/10. 1016/j.jadohealth.2019.04.033

Eisenberg, D., Hunt, J., Speer, N., \& Zivin, K. (2011). Mental health service utilization among college students in the United States. Journal of Nervous and Mental Disease, 199, 301-308. https:// doi.org/10.1097/NMD.0b013e3182175123

Fernández-Aranda, F., Casas, M., Claes, L., Bryan, D. C., Favaro, A., Granero, R., Gudiol, C., Jiménez-Murcia, S., Karwautz, A., Le Grange, D., Menchón, J. M., Tchanturia, K., \& Treasure, J. (2020). COVID-19 and implications for eating disorders. European Eating Disorders Review, 28, 239-245. https://doi.org/10.1002/erv. 2738

Ferster, C. B. (1973). A functional analysis of depression. American Psychologist, 28, 857-870. https://doi.org/10.1037/h0035605

Fitzsimmons-Craft, E. E., Balantekin, K. N., Eichen, D. M., Graham, A. K., Monterubio, G. E., Sadeh-Sharvit, S., Goel, N. J., Flatt, R. E., Saffran, K., Karam, A. M., Firebaugh, M.-L., Trockel, M., BarrTaylor, C., \& Wilfley, D. E. (2019). Screening and offering online programs for eating disorders: Reach, pathology, and differences across eating disorder status groups at 28 US universities. International Journal of Eating Disorders, 52, 1125-1136. https:// doi.org/10.1002/eat.23134

Gallagher, M. W., Smith, L. J., Richardson, A. L., \& Long, L. J. (2021). Examining associations between COVID-19 experiences and posttraumatic stress. Journal of Loss \& Trauma. https://doi.org/10. 1080/15325024.2021.1886799

Garner, D. M., \& Garfinkel, P. E. (1980). Socio-cultural factors in the development of anorexia nervosa. Psychological Medicine, 10, 647-656. https://doi.org/10.1017/s0033291700054945

Graham, A. K., Trockel, M., Weisman, H., Fitzsimmons-Craft, E. E., Balantekin, K. N., Wilfley, D. E., \& Taylor, C. B. (2019). A screening tool for detecting eating disorder risk and diagnostic symptoms among college-age women. Journal of American College Health, 67, 357-366. https://doi.org/10.1080/07448481.2018. 1483936

Grant, K. E., Compas, B. E., Stuhlmacher, A. F., Thurm, A. E., McMahon, S. D., \& Halpert, J. A. (2003). Stressors and child and adolescent psychopathology: Moving from markers to mechanisms of risk. Psychological Bulletin, 129, 447-466. https://doi.org/10. 1037/0033-2909.129.3.447

Jansen, A. (1998). A learning model of binge eating: Cue reactivity and cue exposure. Behaviour Research and Therapy, 36, 257-272. https://doi.org/10.1016/s0005-7967(98)00055-2

Kaparounaki, C. K., Patsali, M. E., Mousa, D. V., Papadopoulou, E. V. K., Papadopoulou, K. K. K., \& Fountoulakis, K. N. (2020). University students' mental health amidst the COVID-19 quarantine in Greece. Psychiatry Research, 290, 113111. https://doi.org/10. 1016/j.psychres.2020.113111

Kessler, R. C., Amminger, G. P., Aguilar-Gaxiola, S., Alonso, J., Lee, S., \& Üstün, T. B. (2007). Age of onset of mental disorders: A review of recent literature. Current Opinion in Psychiatry, 20, 359-364. https://doi.org/10.1097/YCO.0b013e32816ebc8c

Kroenke, K., \& Spitzer, R. L. (2002). The PHQ-9: A new depression diagnostic and severity measure. Psychiatric Annals, 32, 509-515. https://doi.org/10.3928/0048-5713-20020901-06

Lasgaard, M., Goossens, L., Bramsen, R. H., Trillingsgaard, T., \& Elklit, A. (2011). Different sources of loneliness are associated with different forms of psychopathology in adolescence. Journal of Research in Personality, 45, 233-237. https://doi.org/10.1016/j. jrp.2010.12.005

Lê Cook, B., Trinh, N. H., Li, Z., Hou, S. S., \& Progovac, A. M. (2017). Trends in racial-ethnic disparities in access to mental 
health care, 2004-2012. Psychiatric Services, 68, 9-16. https:// doi.org/10.1176/appi.ps.201500453

Lechner, W. V., Lauren, K. R., Patel, S., Grega, C., \& Kenne, D. R. (2020). Changes in alcohol use as a function of psychological distress and social support following COVID-19 related University closings. Addictive Behaviors. https://doi.org/10.1016/j.addbeh. 2020.106527

Lipson, S. K., Phillips, M. V., Winquist, N., Eisenberg, D., \& Lattie, E. G. (2021). Mental health conditions among community college students: A national study of prevalence and use of treatment services. Psychiatric Services. https://doi.org/10.1176/appi. ps. 202000437

Manea, L., Gilbody, S., \& McMillan, D. (2012). Optimal cut-off score for diagnosing depression with the patient health questionnaire (PHQ-9): A meta-analysis. CMAJ, 184, E191-196. https://doi.org/ 10.1503/cmaj. 110829

Moore, M. T., Anderson, N. L., Barnes, J. M., Haigh, E. A. P., \& Fresco, D. M. (2014). Using the GAD-Q-IV to identify generalized anxiety disorder in psychiatric treatment seeking and primary care medical samples. Journal of Anxiety Disorders, 28, 25-30. https://doi.org/10.1016/j.janxdis.2013.10.009

Morin, C. M., Belleville, G., Bélanger, L., \& Ivers, H. (2011). The insomnia severity index: Psychometric indicators to detect insomnia cases and evaluate treatment response. Sleep, 34, 601-608. https://doi.org/10.1093/sleep/34.5.601

Newman, M. G., Holmes, M., Zuellig, A. R., Kachin, K. E., \& Behar, E. (2006). The reliability and validity of the panic disorder selfreport: A new diagnostic screening measure of panic disorder. Psychological Assessment, 18, 49-61. https://doi.org/10.1037/ 1040-3590.18.1.49

Newman, M. G., Zuellig, A. R., Kachin, K. E., Constantino, M. J., Przeworski, A., Erickson, T., \& Cashman-McGrath, L. (2002). Preliminary reliability and validity of the generalized anxiety disorder questionnaire-IV: A revised self-report diagnostic measure of generalized anxiety disorder. Behavior Therapy, 33, 215-233. https://doi.org/10.1016/S0005-7894(02)80026-0

Peltier, M. R., Verplaetse, T. L., Mineur, Y. S., Petrakis, I. L., Cosgrove, K. P., Picciotto, M. R., \& McKee, S. A. (2019). Sex differences in stress-related alcohol use. Neurobiology of Stress, 10, 100149. https://doi.org/10.1016/j.ynstr.2019.100149

Phillipou, A., Meyer, D., Neill, E., Tan, E. J., Toh, W. L., Van Rheenen, T. E., \& Rossell, S. L. (2020). Eating and exercise behaviors in eating disorders and the general population during the COVID-19 pandemic in Australia: Initial results from the COLLATE project. International Journal of Eating Disorders, 53, 1158-1165. https:// doi.org/10.1002/eat.23317

Pittig, A., Treanor, M., LeBeau, R. T., \& Craske, M. G. (2018). The role of associative fear and avoidance learning in anxiety disorders: Gaps and directions for future research. Neuroscience \& Biobehavioral Reviews, 88, 117-140. https://doi.org/10.1016/j. neubiorev.2018.03.015

Prins, A., Ouimette, P., Kimerling, R., Cameron, R. P., Hugelshofer, D. S., Shaw-Hegwer, J., Thrailkill, A., Gusman, F. D., \& Sheikh, J. I. (2003). The primary care PTSD screen (PC-PTSD): Development and operating characteristics. Primary Care Psychiatry, 9, 9-14. https://doi.org/10.1185/135525703125002360

Rodriguez, L. M., Litt, D. M., \& Stewart, S. H. (2020). Drinking to cope with the pandemic: The unique associations of COVID19-related perceived threat and psychological distress to drinking behaviors in American men and women. Addictive Behaviors, 110, 106532. https://doi.org/10.1016/j.addbeh.2020.106532

Sidor, A., \& Rzymski, P. (2020). Dietary choices and habits during COVID-19 lockdown: Experience from Poland. Nutrients, 12(Suppl 6), 1657. https://doi.org/10.3390/nu12061657

Thombs, B. D., Kwakkenbos, L., Levis, A. W., \& Benedetti, A. (2018). Addressing overestimation of the prevalence of depression based on self-report screening questionnaires. CMAJ, 190, E44-E49. https://doi.org/10.1503/cmaj.170691

Touyz, S., Lacey, H., \& Hay, P. (2020). Eating disorders in the time of COVID-19. Journal of Eating Disorders, 8, 19. https://doi.org/10. 1186/s40337-020-00295-3

Twenge, J., \& Joiner, T. E. (2020). Mental distress among US adults during the COVID-19 pandemic. PSsyArXiv. https://doi.org/10. 31234/osf.io/wc8ud

Wang, C., Pan, R., Wan, X., Tan, Y., Xu, L., Ho, C. S., \& Ho, R. C. (2020). Immediate psychological responses and associated factors during the initial stage of the 2019 coronavirus disease (COVID19) epidemic among the general population in China. International Journal of Environmental Research and Public Health. https://doi.org/10.3390/ijerph17051729

Weitzman, E. R., Nelson, T. F., \& Wechsler, H. (2003). Taking up binge drinking in college: The influences of person, social group, and environment. Journal of Adolescent Health, 32, 26-35. https:// doi.org/10.1016/s1054-139x(02)00457-3

World Health Organization. (2020). WHO coronavirus disease (COVID-19) dashboard. Retrieved June 8, 2020, from https:// covid19.who.int/

Publisher's Note Springer Nature remains neutral with regard to jurisdictional claims in published maps and institutional affiliations. 